\title{
Memory training for individuals with Alzheimer's disease improves name recall
}

\author{
J.P. Kesslak, ${ }^{1,3}$ K. Nackoul ${ }^{2}$ and C.A. Sandman ${ }^{2,3}$ \\ Departments of ${ }^{1}$ Neurology and ${ }^{2} P$ sychiatry, and ${ }^{3}$ Institute for Brain Aging, \\ University of California, Invine, CA 92697-4540, USA
}

Correspondence to: J.P. Kesslak at above address

\begin{abstract}
Alzheimer's disease is clinically characterized by a variety of progressive cognitive deficits, most notably an impaired ability to acquire new information, such as name recall. Eleven demented patients and 11 controls participated in a 4 week memory program that included training in name-face recall. Individuals were taught strategies for name-face rehearsal, and administered task specific and standardized tests to assess the intervention efficacy. During the memory training patients improved recall of names and faces $(p<0.05)$, while controls remained stable. Patients also improved on the weekly standardized measures, including the Kendrick Digit Copy and Geriatric Depression Scale $(p<0.05)$. Thus, the memory training can be beneficial for improving name recall and some aspects of behavior. This can provide a framework for development of programs to enhance cognitive function in patients with dementia and may tap biological mechanisms that promote neural plasticity to compensate for the degenerative condition.
\end{abstract}

Keywords: Alzheimer's disease - Behavior - Cognitive rehabilitation - Intervention - Memory

This article originally appeared in a modified form in Alzheimer's Research (1997), 3, 151-157.

\section{INTRODUCTION}

The dementia in Alzheimer's Disease is characterized by quantitative and qualitative deficits in acquisition of new learning (Cummings and Benson, 1992). Difficulty with memory, language, and orientation to time and place progressively decline through the course of dementia to impair activity in daily living skills, and disturb family and social interactions. Difficulty in acquisition and recall of new information results in frustration, depression and withdrawal from interpersonal interactions. Impaired acquisition and recall of new and familiar names is problematic for the patient and can contribute to difficulty with social interactions, increase withdrawal and may be associated with other behavioral symptoms.

However, patients with dementia are not totally amnestic, particularly in the early to moderate stages of the disorder (Cummings and Benson, 1992). Attempts with behavioral strategies have shown marginal benefits to improve memory in patients with dementia; possibly since they stem from retraining methods that focus on language and memory designed for patients with head trauma (van der Linden and van der Kaa, 1989; Berrol, 1990), healthy volunteer (Cermak, 1975; Minninger, 1984), and aged individuals
(Poon et al., 1980; Smith, 1980; Zarit et al., 1981). These strategies encourage semantic or phonemic association to assist in the manipulation and organization of material.

Unfortunately, many cognitive strategies have not been successful with patients with $\mathrm{AD}$, probably because the foundation of these procedures (the ability to make associations) is vulnerable in these patients (Craik and Watkins, 1973; Morris and Kopelman, 1986). Computer based recognition training for surroundings and biography has produced improvement for patients with $A D$, but no generalization (Hofmann et al., 1996). Application of strategies that increase effort, interest and arousal have been found to facilitate recall of names and information related to significant events (Sandman, 1993). However, the ability to generalize the benefits or impact on standardized behavioral measures was not examined. Providing cognitive support during learning and retrieval can also enhance memory function in $\mathrm{AD}$ (Backman, 1996), and can draw on associations related to the to-beremembered material. Training programs that actively involve the patient appear to be the most successful, however benefits eventually deteriorate as the disease 
progresses (Quayhagen et al., 1995). Thus, there is a need to develop behavioral strategies that actively engage the patient and are relevant to the person's daily functions.

In this study, the emphasis for names and faces was placed on increased effort for learning and recall of information, rather than teach sophisticated cognitive strategies that required substantial emotional and intellectual resources. Generalization of training is examined in relation to external measures of memory, attention and mood. In response to the training, the patients with $\mathrm{AD}$ showed increased ability for name recall and general improvement in a set of standard cognitive measures.

\section{METHODS}

\section{Subjects}

Eleven individuals in the early to moderate stages of $\mathrm{AD}$ (mean age $66.36 \pm 6.99$ years) and age matched controls (mean age $64.11 \pm 7.70$ years) were recruited from the UCI Alzheimer's Disease and Aging Clinic and the Memory Disorders Clinic. Individuals volunteered to participate in the study, which was reviewed and approved by the Institutional Review Board. Patients were evaluated and diagnosed for dementia according to NINCDS-ADRDA guidelines for AD (McKhann et al., 1984). Diagnostic evaluation of patients included complete neurological and psychological evaluations including the CERAD test battery. Score on the Mini Mental State Exam was 28 or greater for controls, and 26-14 for patients with AD. Brain imaging was performed using MRI. Routine laboratory tests were also included. Upon completion, diagnosis was made based on the consensus of the clinical staff.

Patients were accompanied in all phases of the interventions by their spouse or caregiver, who received the same instruction and evaluation as the patient, thus acting as a matched control. Although caregivers were not randomly chosen as controls, they were used for comparison since they are exposed to the same environment and conditions, and could reliably monitor the patient's function. The memory training exercises required interaction between the patient and caregiver.

\section{Memory Training Program}

The key concepts stressed in the program for name recall were effort and interest. Task specific tests and standardized cognitive tests were administered each week to assess the performance of participants. Immediate feedback, flexibility in the program design and application were considered critical factors for the success of this program.

Upon entry to the first session, a Polaroid picture was taken of each person, and a photocopy of the pictures was provided for each member. With the sheet in front of them, each participant was instructed to introduce themselves by name and provide information on family, hobbies, interests, accomplishments and other relevant details. All members were encouraged to take notes under the appropriate pictures. After introductions, members were asked to describe other members when asked, not by name, but by hobbies, interests and accomplishments. Errors were immediately corrected.

Patient and caregiver teams were instructed to take the photos and personal information home to review, and to test each other during the week. The group was told that their ability to identify people will be tested on subsequent sessions. On each subsequent session all participants were shown a sheet of photos with the order reorganized. Each person was asked to write the first and last name under the pictures that correspond to the face. After this, verbal recognition was tested in the group. Failures to name members were accompanied with encouragement to recall aspects of the person's hobbies, interests, etc. Name recall was scored immediately, tabulated and graphically presented as feedback to the group. Participants were continually encouraged to practice and rehearse at home.

A brief battery of standard psychometric tests, administered to all participants at the end of each training session, measured attention, cognition and mood (i.e. depression). The 15 min weekly group test battery included: Sustained Attention (cancellation of 2's and 7's), Kendrick Digit Copying, Geriatric Depression Scale (GDS) and the Activities of Daily Living (ADL) from the Blessed Dementia Rating Scale. Tests were administered to both patients and controls. Caregivers were asked to rate the patients' abilities on the ADL from the Blessed Dementia Rating Scale.

Data was analyzed with repeated measures analysis of variance (ANOVA) to determine between group differences, change over time and interactions. Because some measures may not be sensitive to small weekly changes they were examined at the beginning and end of the interventions with a matched-pair t-test.

\section{RESULTS}

\section{Task Specific Measures}

Memory for names was analyzed separately for recall of one name (first or last) and recall of both names. Each person was given a photocopy of the pictures and 
A

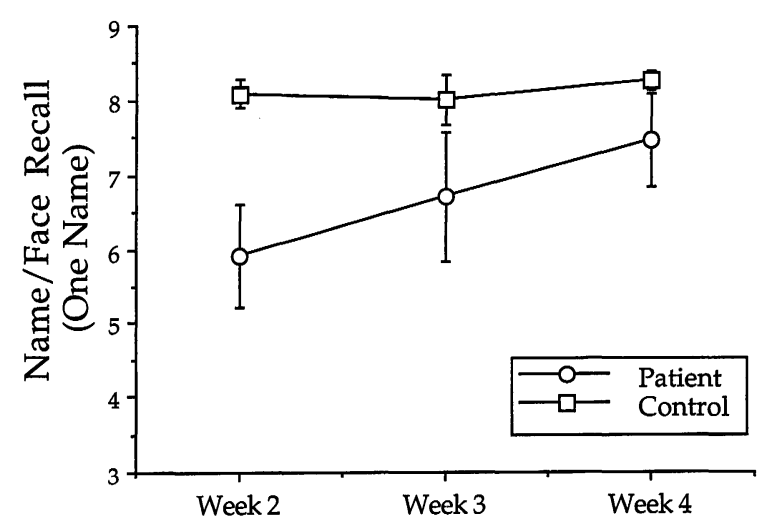

B

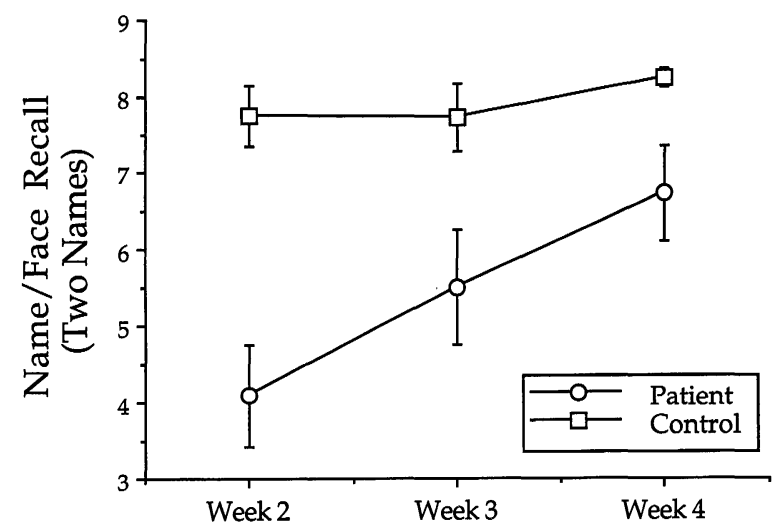

FIG. 1. Recall of either the first or last name (A), or both the first and last name $(B)$ improved across weeks of memory training for the patients with dementia. Agematched controls had learned approximately all eight first and last names of group participants by the second week of memory training and maintained a high level of performance.

asked to name the individuals. Recall of names improved significantly for patients as training progressed (Figure 1) for both a single name, and first and last names. As training progressed in the third and fourth weeks, patients improved for recall of one and two names. Controls were able to identify the majority of individuals by either one or both names throughout the program. Thus, for the recall of one name the significant main effect of days of training $(F(2,38)=4.16$, $p<0.05)$ can be attributed to improvement in the patients' performance, while control performance remained stable. Differential rates of improvement between controls and patients was marginally significant (groups $\times$ days, $F(2,38)=2.94, \quad p=0.06$ ). Throughout training, control subjects recalled significantly more names than patients $(F(1,19)=3.53$, $p=0.03$ ). Healthy controls consistently performed better than patients on recall of two names $(F(1,19)=$ $12.28, p=0.002)$, with improved performance for patients across weeks of testing $(F(2,38)=11.83$, $p=0.001)$ to produce a significant group $\times$ day interaction $(F(2,38)=6.63, p=0.003)$. Between the first and last test training session there was significant improvement of patients' free recall for either one $(p<0.05)$ or both names of an individual $(p<0.05)$. Thus, increased effort that involves rehearsal of names and review of associated information improves memory. It was anticipated that this improved performance may also be reflected in other types of behavior, as measured on the standardized test battery.

\section{Standardized Measures}

Scores on the Kendrick Digit Copy and the GDS, but not 2's and 7's Selective Attention improved for both patients and controls during the memory training. Patient ratings on the ADL from the Blessed Dementia Rating Scale also improved, but ratings of patients by their caregivers did not always agree.

Performance on the Kendrick Digit Copy task, which is sensitive to psychomotor ability, improved significantly during the memory training $(F(3,42)=$ $5.178, p=0.003$ ) (Figure 2A). Controls increased slightly during the initial 2 weeks and stabilized, with performance significantly better than patients $(F(1,16)$ $=5.584, p=0.031$, which was reflected in a significant group $\times$ time interaction $(F(3,42)=2.936$, $p=0.044)$. Patients with $\mathrm{AD}$ showed a significant improvement between the first and last test session $(p<0.05)$, with the most dramatic change between weeks 1 and 2 . If the increased performance was due to a practice effect it was only seen in the $\mathrm{AD}$ group and was not consistent across days of testing. The patients' self-ratings of depression on the GDS were higher (e.g. more depressed) than controls (Figure 2B) and decreased across sessions. This was reflected by a significant decreased depression score for patients $(p<0.05)$, while controls scores did not change $(p>0.10)$. Increased ability to name individuals may promote greater self-esteem, which can be reflected subsequently by an elevated mood. Patients had significantly lower scores on the Selective Attention test than caregivers $(F(1,15)=21.009, \quad p=0.001)$ (Figure 2C). The controls had the largest improvement on the attention task, but neither group 

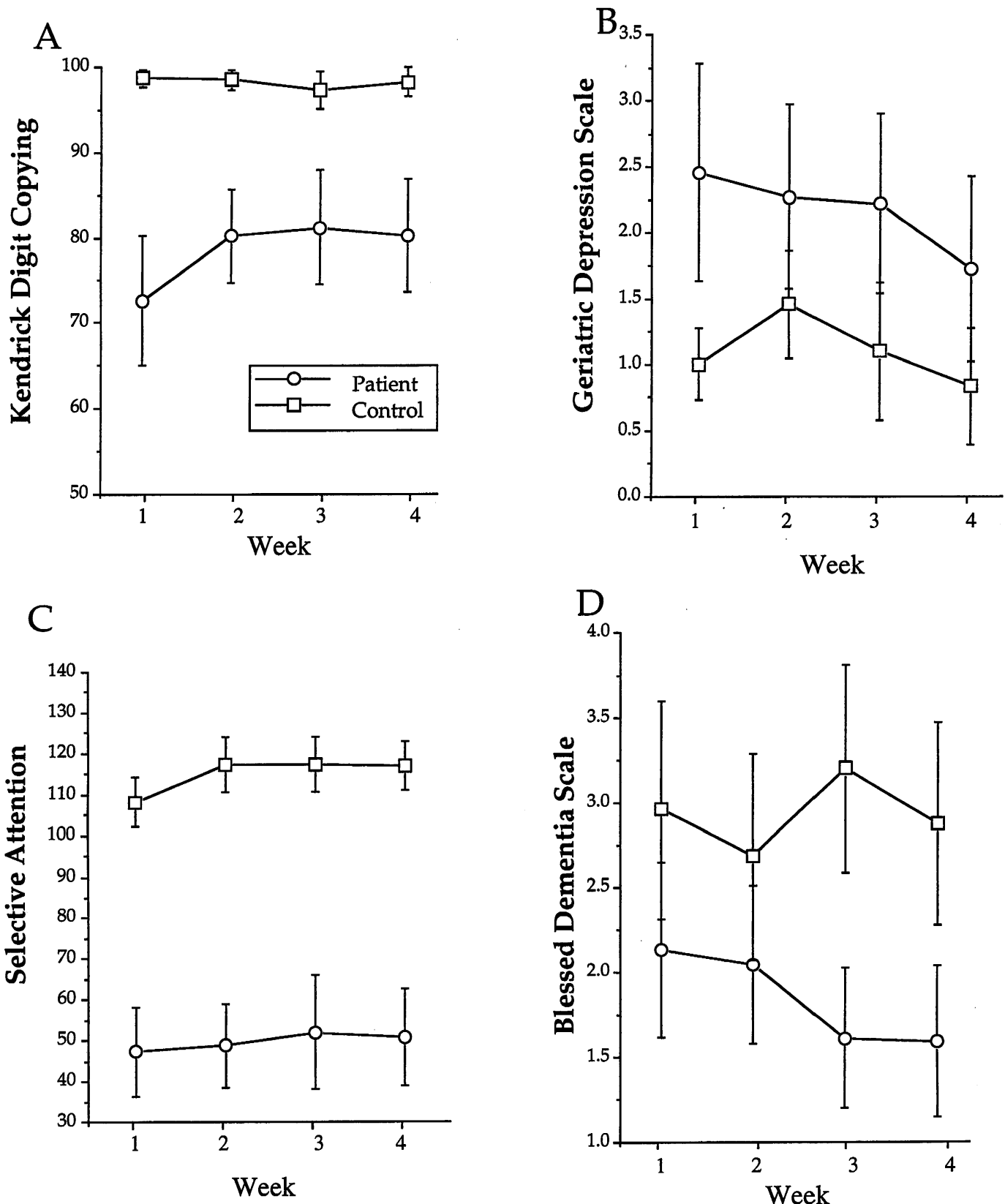

FIG. 2. (A) Patients showed the most dramatic increase on the Kendrick Digit Copying task between weeks 1 and 2 of the memory training. Control performance was stable at a significantly higher level of performance compared with patients. (B) Scores on the Geriatric Depression scale indicated that overall patients rated themselves more depressed than controls, which decreased slightly during the memory training. (C) Both patients and controls showed stable performance on the Selective Attention task across training sessions, with controls having consistently more correct responses than patients. (D) Ratings of activities of daily living from the Blessed Dementia Scale indicated that when patients were given a task that improved performance, caregiver ratings indicated an increase in functional deficits, whereas patients rated themselves as improving.

improved during the memory training session $(F(3,45)$ $=1.12, p=0.351)$. Ratings of patient ADL by their caregivers showed a trend for higher rating (e.g., lower function) than patient self-ratings $(F(1,16)=1.329$, $p=0.266$ ) (Figure 2D). Throughout the course of training sessions the caregivers rated patients more impaired, while patients rated themselves as improving. Patients rated themselves as improved as a result of the memory training $(p<0.05)$, while caregiver ratings of patients did not change $(p>0.10)$. 


\section{DISCUSSION}

Patients with $\mathrm{AD}$ exhibit cognitive deficits that impair recall of names and faces, and daily events. The results of the present study indicate that they can learn new information and their performance can be improved through a memory training program. There are two central findings of this study. First, there is an improvement in task specific activity of improved name-face recall. Second, a modest generalized effect of the intervention was detected with measures of mood and function. Forgetting names and confusing words are among the first and most distressing symptoms of $\mathrm{AD}$ noticed by patients and caregivers (Howard and Patterson, 1989). Our data show that rehearsal of names in association with visual cues (e.g. pictures) and personal characteristics such as interests, hobbies, family etc. can significantly improve the patients' with $\mathrm{AD}$ ability to properly identify another individual.

With the effortful procedures of rehearsal and engagement of 'automatic' processes, the patients with $\mathrm{AD}$ were able to learn and recall the names of new acquaintances. This is consistent with other findings that suggest recall of names and other information can improve during memory training for patients with dementia when increased effort and rehearsal is practiced (Sandman, 1993). By the end of the 4 week training period the patients' ability to recall names was similar to that of controls, who could recall most names by the end of the first session. Integration of material can be facilitated by forming a number of associations or semantic elaboration, with names to elicit proper face identification (Scogin and Prohaska, 1993). Recall of information associated with an individual may promote name recall and act as compensation for inefficient consolidation processes. The chaining of cues to an individual's name provides additional information for encoding and stimulating name recall (Murdock, 1991). Association between names and faces was further strengthened by rehearsal and practice with correction during the training session and at home with the caregiver. The effort associated with practice may be a critical component to the success of this learning strategy. As the neurodegeneration of $\mathrm{AD}$ progresses there are fewer cognitive strategies available for use and they may be less complex, similar to strategies adopted early in development for learning. Just as the most successful approach for a child learning the multiplication tables may be rote memory, the same may apply to the patient with $\mathrm{AD}$ as more complex cognitive processes become compromised.

The increased ability to identify individuals by name may relieve stress that could arise from forgetting names, and facilitate social interactions. Patients rating themselves as less depressed could contribute to this effect. It is apparent from the standardized tests that even though memory for name recall can be enhanced, there are only modest generalized effects of the intervention on behavior. Improved psychomotor speed during the training procedure shows a mild significant effect in the $\mathrm{AD}$ group but not in controls. A portion of the improved speed may be practice effects, however it was not apparent in controls or across all training sessions. Perhaps the most interesting finding was that the patients perceived an increase of their ADLs. This constellation of effects suggests an improved mood, however there was no evidence of stimulating improved higher attentional or cognitive processes. Interestingly, caregivers reported a decrease in patient ADLs. In some instances caregivers reported that the patients, in attempting to assume more responsibility, disrupted daily routine. As a consequence of increased patient improvement, an increased demand may be placed on the caregiver to support the patient's efforts. The dynamics of the interaction between patients and caregivers should be explored further to determine if an effective behavioral intervention places more demands on the caregiver than the expected course of cognitive decline.

In general, behavioral ability was enhanced on tasks related to the training paradigm, but did not generalize, consistent with the cognitive retraining literature (Sohlberg and Mateer, 1989). This may be expected because unlike enrichment in immature organisms, enrichment in adults is event specific (Greenough and Anderson, 1991; Weiler et al., 1995). Neural mechanisms of plasticity continually respond to development, aging, disease and trauma. In the case of disease or trauma the compensatory mechanisms can include neural sprouting to establish new connections and substitution of function, where other brain areas assume the ability to carry out activities of dysfunctional neural circuits (Stein et al., 1983; Cotman and Anderson, 1988). Thus, two mechanisms may occur in response to the memory training to facilitate behavior: (1) synaptogenesis associated with new learning to strengthen existing neural circuits and increase their efficiency, and (2) substitution of function in neural circuits that typically are not highly involved in a behavior to compensate for the progressive degeneration associated with dementia. The goal of our training program is to stimulate activity in a neural circuit to promote system plasticity. Results from animal studies indicate that physical activity (Neeper et al., 1995) and learning (Kesslak et al., 1995) can increase production of neurotrophic factors that enhance neuron survival and promote neurite sprouting. Also, higher levels of 
education and other means of stimulating neural activity appear to be negative risk factors for developing dementia and build a cognitive reserve (Orrell and Sahakian, 1995). Unfortunately, the progressive neural degeneration that occurs in $\mathrm{AD}$ exceeds the CNS capacity to compensate for loss of neurons, ultimately resulting in functional deficits. As pharmacological interventions are developed to retard $\mathrm{AD}$ pathogenesis, behavioral interventions that promote cognitive retraining and stimulate neural reorganization will become increasingly important. Indeed, memory training can, as shown here, be beneficial for improving some aspects of behavior and for providing the initial framework for development of programs to enhance cognitive function in patients with dementia. Further studies are needed to determine other strategies to improve cognitive function in patients with $A D$, to examine larger samples sizes and identify patient characteristics for successful application of behavioral interventions.

\section{CONCLUSION}

One of the earliest symptoms of $\mathrm{AD}$ is memory dysfunction, often characterized by difficulty with the recall of names and acquisition of new information. Traditional mnemonic strategies to improve memory have little success with patients with $\mathrm{AD}$, due to the reliance on more complex cognitive processes. We show recall of names can be improved by a simple strategy of increased effort in name rehearsal and information associated with an individual. In addition to improved name recall, there were modest generalized effects that included an elevated mood and improved ratings in activities of daily living. Such strategies probably stimulate neural activity and may be beneficial to enhance endogenous physiological mechanisms that could retard neural degeneration. Thus, application of behavioral strategies to improve cognitive function in patients with $\mathrm{AD}$ may help to compensate for the dysfunction that occurs in response to the disease pathogenesis.

\section{Acknowledgements}

We would like to thank Drs Rodman Shankle and Malcom Dick for assistance in patient evaluations and referrals, and Dr Carl Cotman for helpful discussion. This work has been supported in part by grants NIA AG05142 and the State of California Department of Health Services 94-20360.

\section{REFERENCES}

Backman L (1996) Acta Neurologica Scandinavia Suppl, 165, 109-113.
Berrol S (1990) Archives of Neurology, 47, 219-220.

Cermak LS (1975) Improving your memory. New York: W W Norton.

Cotman CW and Anderson KJ (1988) Advanced Neurology, 47, 313-335.

Craik FI and Watkins MJ (1973) Journal of Verbal Learning Verbal Behaviour, 12, 599-607.

Cummings JF and Benson DF (1992) Dementia: a clinical approach (2nd ed.). Stoneham, Massachussetts: Butterworth-Heinemann.

Greenough WT and Anderson BJ (1991) Annals of the New York Academy of Sciences, 627, 231-247.

Hofmann M, Hock C and Muller-Spahn F (1996) Annals of the New York Academy of Sciences, 777, 249-254.

Howard D and Patterson K (1989) Models for therapy. In: Seron X and Deloche G, eds. Cognitive approaches in neuropsychological rehabilitation. Hillsdale, New Jersey: Lawrence Erlbaum Ass.: 39-64.

Kesslak JP, Gomez-Pinilla F, So V, et al. (1995) Social Neuroscience Abstracts, 21, 23.9.

McKhann G, Drachman D, Folstein M et al. (1984) Neurology 34, 939-944.

Minninger J (1984) Total Recall-How to Boost Your Memory Power. Philadelphia: Rodale Press.

Morris RG and Kopelman MD (1986) Quarterly Journal of Experimental Psychiatry, 38, 575-602.

Murdock BB (1991) Serial organization in a distributed memory model. In: Healy AF, Kosslyn SM and Shiffrin RM, eds. Essays in honor of William K Estes. Hilldsdale, New Jersey: Lawrence Erlbaum Ass.: 201-225.

Neeper SK, Gomez-Pinilla F, Choi J et al. (1995) Nature 373, 109.

Orrell M and Sahakian B (1995) British Medical Journal, 310, 951-952.

Poon LW, Fozard JL and Treat NJ (1980) Experimental Aging Research, 4, 235-253.

Quayhagen MP, Quayhagen M, Corbeil RR et al. (1995) Nursing Research, 44, 153-159.

Sandman CA (1993) Clinical Gerontology, 13, 19-33.

Scogin F and Prohaska M (1993) Aiding older adults with memory complaints. Sarasota, Florida: Professional Resource Press.

Smith AD (1980) Age differences in encoding, storage and retrieval. In: Poon LW, Fozard JL, Cermak LS et al., eds. New directions in memory and aging. Hillsdale, New Jersey: Erlbaum: 23-46.

Sohlberg MM and Mateer CA (1989) Journal of Clinical Experimental Neuropsychology, 11, 871-891.

Stein DG, Finger S and Hart T (1983) Behavoural Neural Biology 37, 185-222.

van der Linden M and van der Kaa MA (1989) Reorganization therapy for memory impairments. In: Seron X and Deloche G, eds. Cognitive approaches in neuropsychological rehabilitation. Hillsdale, New Jersey: Lawrence Erlbaum Ass.: 105-158.

Weiler IJ, Hawrylak N and Greenough WT (1995) Behaviour and Brain Research, 66, 1-6.

Zarit SH, Cole KD and Guider GL (1981) Gerontology, 21, 158-164.

(Received 18 June 1997; accepted as revised 3 July 1997) 


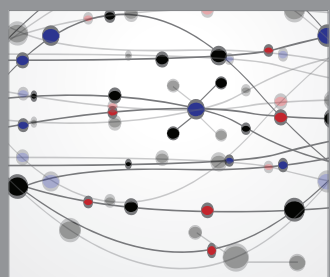

The Scientific World Journal
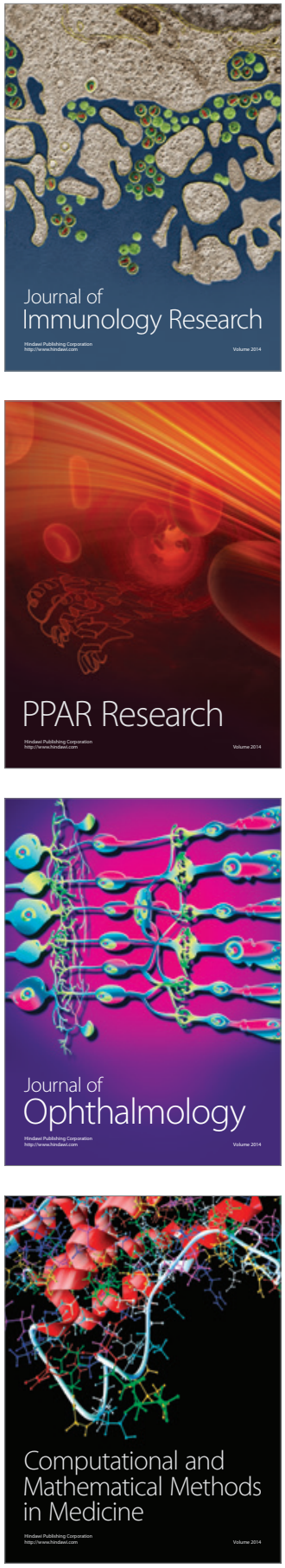

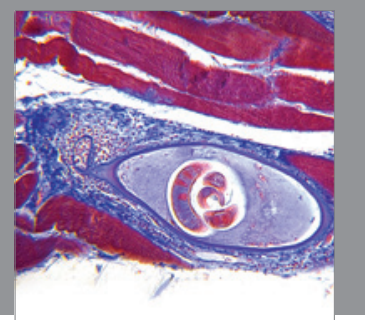

Gastroenterology

Research and Practice
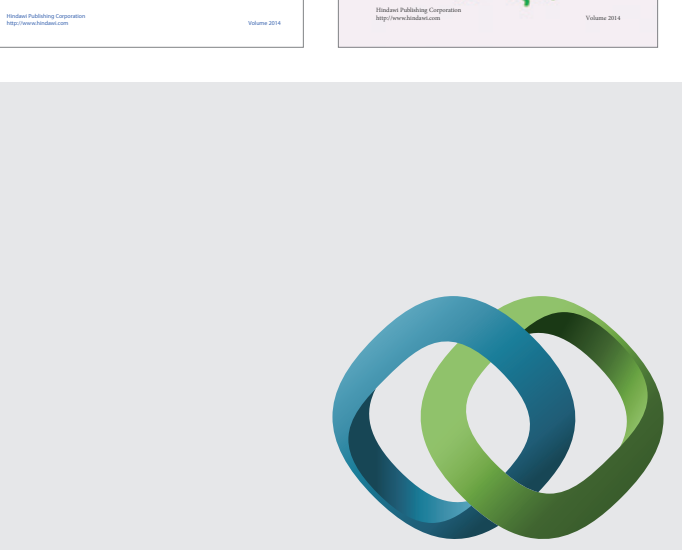

\section{Hindawi}

Submit your manuscripts at

http://www.hindawi.com
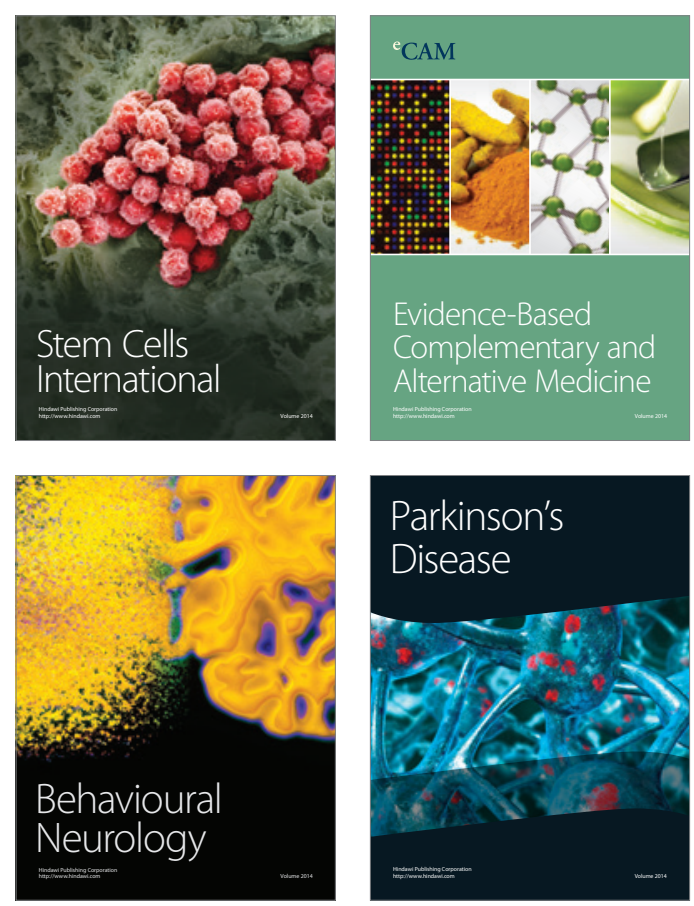

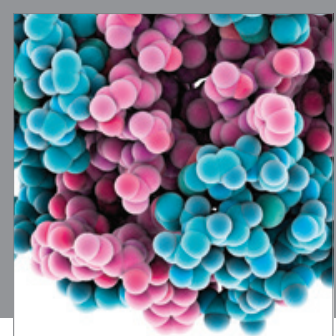

Journal of
Diabetes Research

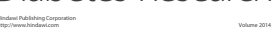

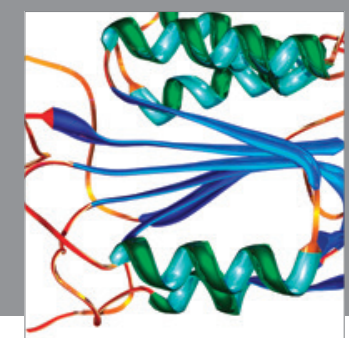

Disease Markers
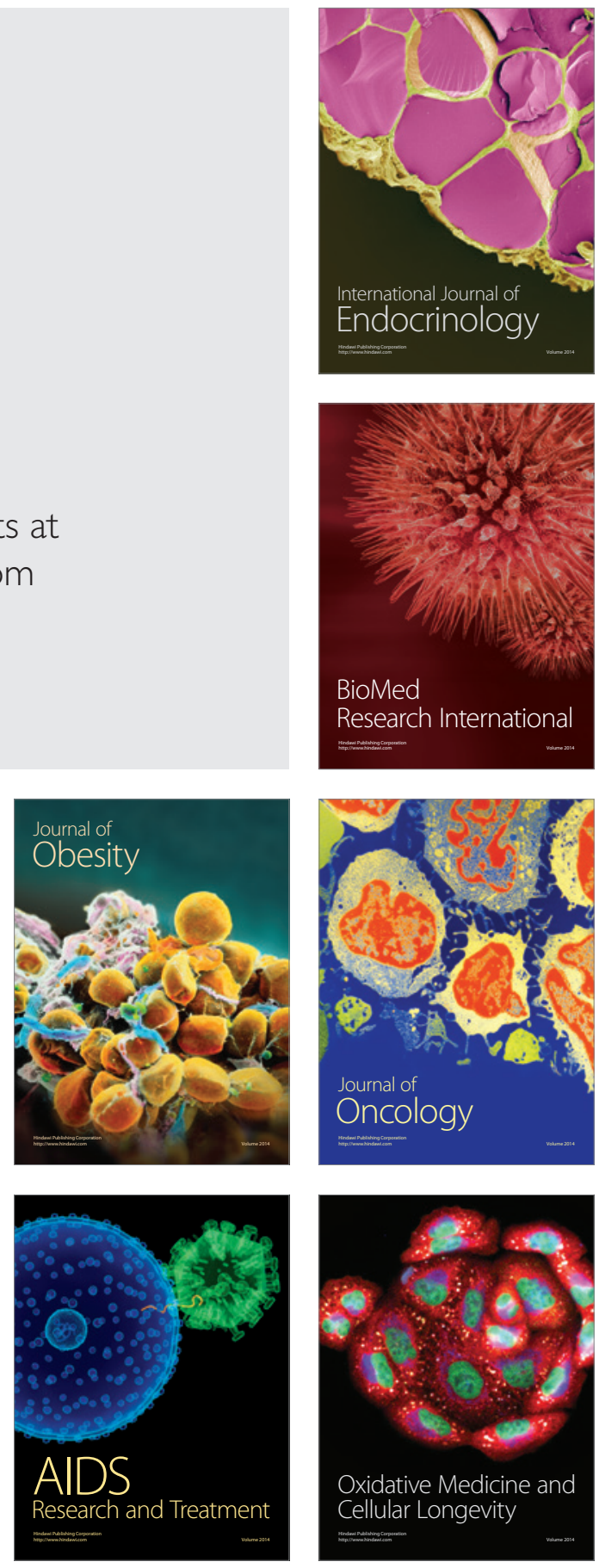